Ricardo Bianconi, Universidade de São Paulo - Instituto de Matemática e Estatística - Caixa Postal 66 281, CEP 05315-970, São Paulo-SP-Brazil, e-mail:bianconi@ime.usp.br

\title{
ON THE CONVERGENCE OF THE INTEGRALS OF A TRUNCATED HENSTOCK-KURZWEIL INTEGRABLE FUNCTION
}

\begin{abstract}
We deal with two ways to truncate a Henstock-Kurzweil integrable function and the convergence of their integrals. We also give an example to show the limitations to the convergence theorem.
\end{abstract}

The problem we deal with here is the following. Let $f:[a, b] \longrightarrow \mathbb{R}$ be a measurable function. For $M$ and $N$ positive real numbers, we define two truncations of $f$ :

$$
\begin{aligned}
& f_{M, N}(x)= \begin{cases}f(x) & \text { if }-N \leq f(x) \leq M \\
M & \text { if } f(x) \geq M \\
-N & \text { if }-N \geq f(x)\end{cases} \\
& \tilde{f}_{M, N}(x)= \begin{cases}f(x) & \text { if }-N \leq f(x) \leq M \\
0 & \text { if } f(x) \geq M \\
0 & \text { if }-N \geq f(x)\end{cases}
\end{aligned}
$$

If $M, N$ go to infinity both $f_{M, N}$ and $\tilde{f}_{M, N}$ converge pointwise to $f$. So if $f$ is Lebesgue integrable, by the Dominated Convergence Theorem, their integrals converge to that of $f$. In particular if we take $M=N$. But this does not happen in the case of the Henstock-Kurzweil integral. In [2, Section 18, pp. 114-118] there is a study of the cases when $\int \tilde{f}_{M, M}$ converges to $\int f$. The

Key Words: convergence, Henstock-Kurzweil integral, truncation

Mathematical Reviews subject classification: 26A39

Received by the editors September 16, 1996 
class of such functions (called HL in [2]) contains properly the Lebesgue integrable functions but there are Henstock-Kurzweil integrable functions outside this class. (See [2, Example 18.3, p. 115].) We give here conditions on the truncations of the form $f_{M, N}$, such that the integrals of the truncated functions converge to the integral of the function. We also give an example showing that there is no such result for truncations of the form $\tilde{f}_{M, N}$.

More precisely, we prove here the following result.

Theorem 1. Let $f:[a, b] \longrightarrow \mathbb{R}$. The following statements are equivalent:

1. $f$ is Henstock-Kurzweil integrable and $\int_{a}^{b} f=A$;

2. $f$ is measurable and there are increasing sequences of positive integers $M_{k}, N_{k}$ and a decreasing sequence of gauges $\delta_{k}$ such that for all $k$ and all $\delta_{k}$-fine partition $P,\left|S(f, P)-S\left(f_{k}, P\right)\right|<1 / k$ and $\left|S\left(f_{k}, P\right)-A\right|<1 / k$, where $f_{k}=f_{M_{k}, N_{k}}$. Moreover, in this case, $\int_{a}^{b} f=A$ and $\int_{a}^{b} f_{k} \longrightarrow \int_{a}^{b} f$, as $k \longrightarrow \infty$.

Proof. We prove first that (2) implies (1). Let $f_{k}$ and $\delta_{k}$ be as in (2). Let $m \leq n$, and $P$ be a $\delta_{n}$-fine partition. Since $\delta_{n} \leq \delta_{m}$, then $P$ is also $\delta_{m}$-fine. Thus

$$
\begin{aligned}
\left|\int_{a}^{b} f_{n}-\int_{a}^{b} f_{m}\right| \leq \mid & \left|\int_{a}^{b} f_{n}-S\left(f_{n}, P\right)\right|+\left|S\left(f_{n}, P\right)-S(f, P)\right| \\
& +\left|S(f, P)-S\left(f_{m}, P\right)\right|+\left|S\left(f_{m}, P\right)-\int_{a}^{b} f_{m}\right|<4 / m .
\end{aligned}
$$

This means that the integrals $\int_{a}^{b} f_{k}$ form a Cauchy sequence and so converge to some $A \in \mathbb{R}$. Given $\varepsilon>0$, choose $n$ such that $\left|\int_{a}^{b} f_{n}-A\right|<\varepsilon / 3$ and $1 / n<\varepsilon / 3$. Then for all $\delta_{n}$-fine partition $P$ we have

$$
|S(f, P)-A| \leq\left|S(f, P)-S\left(f_{n}, P\right)\right|+\left|S\left(f_{n}, P\right)-\int_{a}^{b} f_{n}\right|+\left|\int_{a}^{b} f_{n}-A\right|<\varepsilon .
$$

That is, $f$ is integrable to $A$ and the integrals $\int_{a}^{b} f_{n}$ converge to $\int_{a}^{b} f$.

Now we prove that (1) implies (2). It is known that if $f$ is HenstockKurzweil integrable then it is measurable (see [2, Section 5]). If $|f|$ is also integrable then it is Lebesgue integrable so the Dominated Convergence Theorem gives the desired result. So we can assume that $|f|$ is not integrable. It follows that neither $f^{+}=\max (f, 0)$ nor $f^{-}=\max (-f, 0)$ is integrable.

For each $M, N, \min \left(f^{+}, M\right)$ and $\min \left(f^{-}, N\right)$ are integrable. But their 
integrals go to infinity as $M$ and $N$ go to infinity. On the other hand

$$
\begin{aligned}
\lim _{M \longrightarrow \infty} & \int_{a}^{b}\left(\min \left(f^{+}, M+1\right)-\min \left(f^{+}, M\right)\right) \\
& =\lim _{N \longrightarrow \infty} \int_{a}^{b}\left(\min \left(f^{-}, N+1\right)-\min \left(f^{-}, N\right)\right)=0,
\end{aligned}
$$

because the Lebesgue measure of the sets $\left\{x \in[a, b]: N \leq f^{+}(x) \leq N+1\right\}$ and $\left\{x \in[a, b]: N \leq f^{-}(x) \leq N+1\right\}$ tend to zero as $N$ tend to infinity.

Recall that $f_{M, N}=\min \left(f^{+}, M\right)-\min \left(f^{-}, N\right)$. We show now that we can find increasing sequences of integers $M_{k}, N_{k}$ such that, for all $k, \mid \int_{a}^{b} f_{M_{k}, N_{k}}-$ $\int_{a}^{b} f \mid<1 / 3 k$.

Let $r_{n}=\int_{a}^{b}\left(\min \left(f^{+}, n+1\right)-\min \left(f^{+}, n\right)\right) \geq 0$ and $s_{n}=\int_{a}^{b}\left(\min \left(f^{-}, n+\right.\right.$ 1) $\left.-\min \left(f^{-}, n\right)\right) \geq 0$. By the hypothesis on $f$, and the above, both $r_{n}$ and $s_{n}$ tend to zero as $n$ goes to infinity, and both series $\sum r_{n}$ and $\sum s_{n}$ diverge; also $\int_{a}^{b} \min \left(f^{+}, N\right)=\sum_{0}^{N} r_{n}$, and $\int_{a}^{b} \min \left(f^{-}, N\right)=\sum_{0}^{N} s_{n}$. So, given $\varepsilon>0$, there is $n_{0} \in \mathbb{N}$ such that, for all $n \geq n_{0}, 0 \leq r_{n}, s_{n}<\varepsilon$. Put $R_{N}=\sum_{n=0}^{N} r_{n}$ and $S_{N}=\sum_{n=0}^{N} s_{n}$, and $A=\int_{a}^{b} f$.

Either $R_{n_{0}}-S_{n_{0}}<A$ or $R_{n_{0}}-S_{n_{0}} \geq A$ can happen. In the first case, choose the first integer $n_{1} \geq n_{0}$ such that $R_{n_{1}}-S_{n_{0}} \geq A$. We have that $n_{1}>n_{0}$, and $R_{n_{1}-1}-S_{n_{0}}<A \leq R_{n_{1}}-S_{n_{0}}$. Therefore $0 \leq\left(R_{n_{1}}-S_{n_{0}}\right)-A<r_{n_{1}}<\varepsilon$. Letting $M=n_{1}$ and $N=n_{0}$, we have that $\left|\int_{a}^{b} f_{M, N}-\int_{a}^{b} f\right|<\varepsilon$. With the same type of argument, we can treat the case where $R_{n_{0}}-S_{n_{0}} \geq A$. Now it is a matter of applying this to $\varepsilon=1 / 3 k$.

Put $f_{k}=f_{M_{k}, N_{k}}$. Let $\delta_{k}$ be a decreasing sequence of gauges such that, for all $k$ and for all $\delta_{k}$-fine partition $P,\left|S(f, P)-\int_{a}^{b} f\right|<1 / 3 k$, and $\mid S\left(f_{k}, P\right)-$ $\int_{a}^{b} f_{k} \mid<1 / 3 k$. Then, for all $k$ and all $\delta_{k}$-fine $P$,

$$
\begin{aligned}
\mid S\left(f_{k}, P\right) & -S(f, P) \mid \\
& \leq\left|S(f, P)-\int_{a}^{b} f\right|+\left|\int_{a}^{b} f-\int_{a}^{b} f_{k}\right|+\left|S\left(f_{k}, P\right)-\int_{a}^{b} f_{k}\right| \leq \frac{1}{k},
\end{aligned}
$$

which proves (2).

One could conjecture that the same would be true for the $\tilde{f}_{M, N}$. But the following example shows that this is far from true.

Let $f, g:[-2,2] \longrightarrow \mathbb{R}$ be defined by: 


$$
f(x)=\left\{\begin{aligned}
\frac{2^{k}}{k} & \text { if } x \in\left(a_{j}+\frac{1}{2^{(k-1)}}+\frac{1}{2^{k}}, a_{j}+\frac{1}{2^{(k-2)}}\right) \\
-\frac{2^{k}}{k} & \text { if } x \in\left(a_{j}+\frac{1}{2^{(k-1)}}, a_{j}+\frac{1}{2^{(k-1)}}+\frac{1}{2^{k}}\right) \\
0 & \text { otherwise }
\end{aligned}\right.
$$

and

$$
g(x)=\left\{\begin{aligned}
\frac{2^{(k+1)}}{k+1} & \text { if } x \in\left(a_{j}+\frac{1}{2^{(k-2)}}-\frac{k+1}{k 2^{(k+1)}}, a_{j}+\frac{1}{2^{(k-2)}}\right) \\
-\frac{2^{k}}{k} & \text { if } x \in\left(a_{j}+\frac{1}{2^{(k-1)}}, a_{j}+\frac{1}{2^{(k-1)}}+\frac{1}{2^{k}}\right) \\
0 & \text { otherwise }
\end{aligned}\right.
$$

where $a_{0}=0, a_{j+1}=a_{j}-1 / 2^{j}$ and $k \geq j+1$. Let $h:[-2,6] \longrightarrow \mathbb{R}$ be such that $h(x)=f(x)$ if $x \in[-2,2]$ and $h(x)=g(x-4)$ if $x \in] 2,6]$.

Notice that $f$ is improper Riemann integrable in each interval $\left[a_{j+1}, a_{j}\right]$ and $\int_{a_{j+1}}^{a_{j}} f=0$; and the same is true with $g$ replaced for $f$. Then, for each $u \in(-2,6), h$ is integrable in $[u, 6]$, and $\lim _{u \rightarrow-2} \int_{u}^{6} h=0$. So $h$ is integrable in $[-2,6]$ and $\int_{-2}^{6} h=0$ (by $[2$, Corollary 7.10]).

But let $B_{k}$ be the set where $h(x)=2^{k} / k$ and $C_{k}$ the set where $h(x)=$ $-2^{k} / k$, and $b_{k}=2^{k} m\left(B_{k}\right) / k=\int_{B_{k}} h, c_{k}=-2^{k} m\left(C_{k}\right) / k=\int_{C_{k}} h$.

Notice that $h(x)=2^{k+1} /(k+1)$ for all $x$ in the intervals $\left(a_{j}+1 / 2^{k}+\right.$ $\left.1 / 2^{k+1}, a_{j}+1 / 2^{(k-1)}\right)$, and in the intervals $\left(4+a_{j}+1 / 2^{k-2}-(k+1) / k 2^{k+1}, 4+\right.$ $\left.a_{j}+1 / 2^{k-2}\right), 0 \leq j<k+1$, so $b_{k+1}=2, k \geq 1$. (There are $2 k$ such intervals and the integral of $h$ in each of these intervals is $1 / k$.) And $h(x)=2^{k} / k=2$, for $k=1$, in the interval $(3 / 2,2)$, giving $b_{1}=1$. Similarly we can see that $c_{n}=-2$, for all $n \geq 1$.

Thus any truncation of $h$ of the form $\tilde{h}_{M, N}$ would have integrals given by sums of some $b_{n}$ and $c_{n}$ (including $b_{1}=1$ ) resulting in an odd integer. These integrals will never be close to zero. So the best result known to me in this direction is Lu's Lemma, [3, Lemma 2].

Acknowledgements. The author wishes to thank Professors Chaim S. Hönig, João Carlos Prandini and Severino Toscano do Rego Melo for fruitful conversations. The author also thanks the referee for the questions which helped to clarify some points of this paper. 


\section{References}

[1] R. G. Bartle, A convergence theorem for generalized Riemann integrals, Real Analysis Exchange, Vol. 20(1) (1994-1995), 119-124.

[2] Lee Peng-Yee,Lanzhou lectures on Henstock integration, Series in Real Analysis Volume 2, World Scientific, Singapore, 1989.

[3] Lu Shi-Pan and Lee Peng-Yee, Globally small Riemann sums and the Henstock integral, Real Analysis Exchange, Vol. 16(1990-1991), 537-545. 